\title{
A Fast Image Segmentation Strategy for Measurement Point of Machinery Blade
}

\author{
Jiande Zhanga ${ }^{\text {a }}$ Wei Huang, Lizhong Tu and Qing Yang \\ School of Computer Engineering, Nanjing Institute of Technology, Nanjing 211167, China; \\ azhangjiandexy@163.com
}

Keywords: Image segmentation, Machinery blade, Edge detection, Fast segmentation.

\begin{abstract}
To solve the problem of long time spent on measurement point segmentation and low measurement efficiency in the process of machinery blade measurement, a new fast segmentation algorithm based on edge detection is researched in this paper. Before the segmentation of measurement points, the edge of blade image is detected by a suitable edge detection operator which is defined by analyzing image characteristics, and then the processing blade image is the basement for calculating the threshold value. Numerical experiments show that, the strategy researched in this paper can maintain specific information of blade edge image as well as reduce amount of segmentation information, and the segmentation efficiency of machinery blade image is improved.
\end{abstract}

\section{Introduction}

Image Segmentation of measurement point is one of the most important part for image measurement of machinery blade, and the segmentation results provide a good foundation for future image process. It is a difficult problem of segmentation because of the special characteristics of machinery blade image, such as: complex image gathering environment, various surface defects and so on. Most of the common segmentation strategies are unable to satisfy detection requirements of measurement point image during industrial production. Therefore, there are still a lot of research space to solve this problem.

Based on above problems, a fast image segmentation strategy based on edge detection algorithm and maximum between-cluster variance threshold segmentation algorithm is proposed in this paper. Before measurement point segmentation, a fast edge detection operator will be used to process blade image and a threshold can be determined rapidly based on the detection results.

The structure of the thesis is described as follows: the introduction is given in Section 1. In Section 2 , the strategies of fast measurement point segmentation algorithm is discussed in details, and computer implementation procedure of the new strategies for machinery blade has been presented in Section 3. Numerical experiments results using the new algorithm are presented and conclusions are addressed in the fifth Section.

\section{Fast Image Segmentation Strategy for Measurement Points}

Threshold segmentation which takes global threshold as criteria range is a simple and effective image segmentation strategy, but it usually misses edge information of machinery blade image. Segment machinery blade image based on edge detected image is a good choice to improve segmentation precise and efficiency. So, determine a suitable edge detection operator is the first step. Supposed $f(x, y)$ express any of those pixel in machinery blade image, then the derivative of $x$ direction and y direction can be described as formula (1):

$$
f_{x}(x, y)=\frac{\partial f(x, y)}{\partial x} \quad f_{y}(x, y)=\frac{\partial f(x, y)}{\partial y}
$$

So, the second derivative can be calculated out as follows: 


$$
f_{x x}(x, y)=\frac{\partial^{2} f(x, y)}{\partial x^{2}} \quad f_{y y}(x, y)=\frac{\partial^{2} f(x, y)}{\partial y^{2}} \quad f_{x y}(x, y)=\frac{\partial^{2} f(x, y)}{\partial x \partial y}
$$

Based on the discussion above, we can choose Robert edge detection operator to detect the edge of machinery blade image. Robert edge detection operator takes into account both convex curve characteristics and symmetries of blade edge.

During the process of image segmentation, an average threshold is calculated out to divide the edge detected image into two parts: one including measurement points while another including background of machinery blade image. Specifically, the machinery blade segmented image $g(x, y)$ is described as formula(3):

$$
g(x, y)=\left\{\begin{array}{lc}
Z_{E} & f(x, y) \in Z \\
Z_{B} & \text { other }
\end{array}\right.
$$

Where, $\mathrm{f}(\mathrm{x}, \mathrm{y})$ is the original image, $\mathrm{Z}$ is a gray level set within the whole image gray level range and its value is in the interval of $\left(\mathrm{Z}_{1}, \mathrm{Z}_{\mathrm{k}}\right) . \mathrm{Z}_{\mathrm{E}}$ and $\mathrm{Z}_{\mathrm{B}}$ are respectively the target and background gray level.

\section{Computer Implementation of Fast Image Segmentation}

The fast algorithm can be applied to the machinery blade image segmentation. During the segmentation, there are two parts: edge detection with Robert edge detection operator and machinery blade image segmentation. The procedure can be described as follows:

Step 1. Read original machinery blade image pixel-by-pixel, and save the row, column and grayscale values of each pixel into a matrix, then a machinery blade image space is constructed.

Step 2. Determine edge detection operator. Supposed that, the Robert operator is $g(x, y)$ and $f(x, y)$ means the gray value of the point $(\mathrm{x}, \mathrm{y})$, the formula is defined as below:

$$
g(x, y)=\sqrt{[f(x, y)-f(x+1, y+1)]^{2}+[f(x, y+1)-f(x+1, y)]^{2}}
$$

The approximate formula is:

$$
g(x, y)=|f(x, y)-f(x+1, y+1)|+|f(x, y+1)-f(x+1, y)|
$$

The edge detection template is:

$$
\left[\begin{array}{cc}
1 & 0 \\
0 & -1
\end{array}\right] \quad\left[\begin{array}{cc}
0 & 1 \\
-1 & 0
\end{array}\right]
$$

Based on the template, we can get an edge detected machinery blade image.

Step 3. Calculate global average threshold. Read machinery blade image pixel by pixel and compute the total number in the image:

$$
\text { TotalNum }=\sum_{i=0}^{255} m_{i}
$$

Where, mi means number of pixel whose gray value is $i$. Then, a global weighted mean value of the image can be computed as below:

$$
\text { avgTotal }=\frac{\sum_{i=0}^{255} i * m_{i}}{\text { TotalNum }}
$$

Supposed that, the local weighted mean gray value of the two parts divided by edge detection algorithm is respectively avgTotal1 and avgTotal2. So the global average threshold value is:

$$
T_{1}=\operatorname{avgTotal}_{1} * \frac{M_{1}}{\text { TotalNum }_{\text {avgTotal }}} * \frac{M_{2}}{\text { TotalNum }}
$$

Step 4. Calculate maximum between-cluster variance. Take $\mathrm{T} 1$ as a threshold to segment machinery blade image, probabilities of pixels distributed in the two regions can be calculated out as follows: 
$\omega_{1}=\sum_{i=0}^{t} p_{i} \quad \omega_{2}=\sum_{i=t+1}^{255} p_{i}$

Formulas to calculate mean values are:

$\mu_{1}=\frac{\sum_{i=0}^{t} i^{*} p_{i}}{\omega_{1}} \quad \mu_{2}=\frac{\sum_{i=t+1}^{255} i^{*} p_{i}}{\omega_{2}}$

So, the total average mean value can be calculated out as below:

$\mu(t)=\omega_{1} * \mu_{1}(t)+\omega_{2} * \mu_{2}(t)$

We can get maximum between-cluster variance by formula (13):

$\sigma^{2}(t)=\omega_{1} *\left(\mu_{1}-\mu\right)^{2}+\omega_{2} *\left(\mu_{2}-\mu\right)^{2}$

Finally, the machinery blade image can be segmented by the algorithm.

\section{Numerical Experiment}

The classical machinery blade image is segmented in the experiment to illustrate the validation of algorithm researched in this paper. The experiments results of maximum variance method and fast segmentation method are respectively shown in table 1 and figure 1 .

Table 1. Experiments results

\begin{tabular}{cc}
\hline Method & Segmentation time(s) \\
\hline Maximum variance & 0.635 \\
Fast segmentation & 0.416 \\
\hline
\end{tabular}
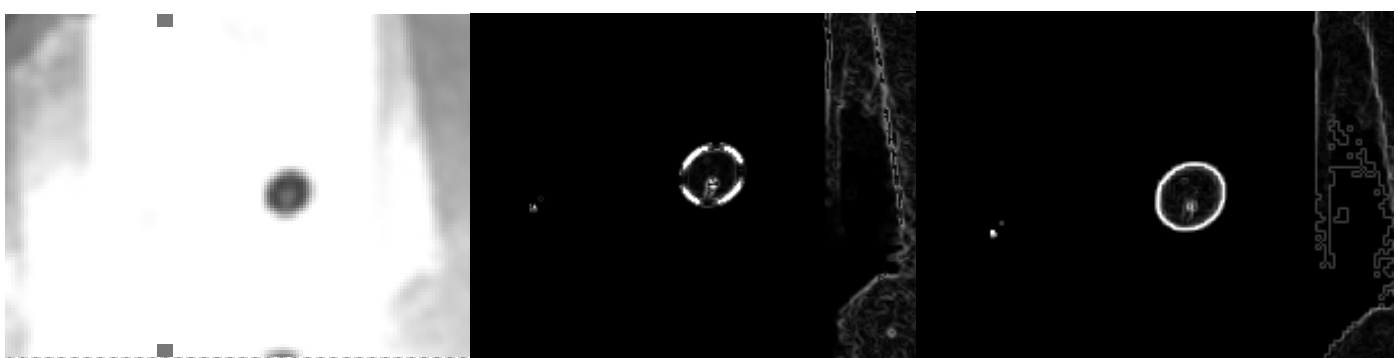

Fig.1 Measurement point image segmentation results

From table 1 we can see that, the fast segmentation method researched in this paper tends to be more efficient than the classic maximum variance method. In figure 1, image in the left is he original point image of machinery blade, the middle image is the result segmented by maximum variance algorithm, and the right image is the result segmented by the algorithm researched in this paper. It is plain to see that the researched model performs better.

\section{Conclusion}

According to the characteristics of the machinery blade image, a fast segmentation method combining edge detection and maximum variance strategy is researched in this paper. From experiment result, we can see that, the fast segmentation strategy researched in this paper spends less time and get a better segmentation result. The algorithm presented is an effective and competitive method for the measurement point segmentation for machinery blade image.

\section{Acknowledgements}

This work is supported in part by the Natural Scientific Research Foundation of JiangSu Province (Grant No.BK20150731), Natural Scientific Research Foundation of Colleges in JiangSu Province 
(Grant No.15KJB520014) and Scientific Research Foundation of Nanjing Institute of Technology (Grant No.CKJA201306). We are grateful to express our thanks.

\section{References}

[1]. S.Arumugadevi, V.Seenivasagam. Color Image Segmentation Using Feedforward Neural Networks with FCM. International Journal of Automation and Computing.Vol.13(2016) No.5, p. 491-500.

[2]. Chengzhang Zhu, Beiji Zou, Yao Xiang, et al. An Ensemble Retinal Vessel Segmentation Based on Supervised Learning in Fundus Images. Chinese Journal of Electronics. Vol.25 (2016), No.3, p.503-511.

[3]. Soumen Biswas, Dibyendu Ghoshal, Ranjay Hazra. A new algorithm of Image Segmentation using Curve Fitting Based Higher Order Polynomial Smoothing. International Journal for Light and Electron Optics. Vol127 (2016) No.20, p.8916-8925.

[4]. R.S.Medeiros,J. Scharcanski,A.Wong. Image segmentation via multi-scale stochastic regional texture appearance models. Computer Vision and Image Understanding.Vol.142 (2015)No.C, p.23-36.

[5]. He Mingge, Yin Ying, Yin Xiangyun, et al. Edge detection of magnetic tile cracks, based on wavelet. High Technology Letters.Vol.21 (2015) No.3, p.276-281.

[6]. Kit Yan Chan,Ulrich EngelkebUlrich,Nimsiri Abhayasinghe. An Edge Detection Framework Conjoining with IMU Data for Assisting Indoor Navigation of Visually Impaired Persons. Expert Systems with Applications. Vol.67 (2016) No.1, p.272-284.

[7]. Sasirooba Thirumavalavann,Sasikala Jayaraman. An improved teaching-learning based robust edge detection algorithm for noisy images. Journal of Advanced Research. Vol.7 (2016) No.6, p.979-989.

[8]. Mayur A. Ingle, Girish R. Talmale. Respiratory Mask Selection and Leakage Detection System Based on Canny Edge Detection Operator. Procedia Computer Science. Vol.78 (2016) No.1, p.323-329.

[9]. Hongyoon Choi, Kyong Hwan Jin. Fast and robust segmentation of the striatum using deep convolutional neural networks. Journal of Neuroscience Methods. Vol.274 (2016) No.1, p.146-153.

[10]. Jennifer Alven,Alexander Norlen,Olof Enqvist, et al. Fast and Robust Registration for Multi-Atlas Segmentation. Pattern Recognition Letters, Vol.80 (2016) No.1, p.249-255. 